\title{
Odako Growth of Dense Arrays of Single-Walled Carbon Nanotubes Attached to Carbon Surfaces
}

\author{
Cary L. Pint ${ }^{1,3}$, Noe T. Alvarez ${ }^{2,3}$, and Robert H. Hauge ${ }^{2,3}(\bowtie)$ \\ ${ }^{1}$ Department of Physics and Astronomy, ${ }^{2}$ Department of Chemistry, ${ }^{3}$ Richard E. Smalley Institute for Nanoscale Science \\ and Technology, Rice University, Houston, TX 77005, USA \\ Received: 13 March 2009 / Revised: 14 April 2009 / Accepted: 14 April 2009 \\ (C) Tsinghua University Press and Springer-Verlag 2009. This article is published with open access at Springerlink.com
}

\begin{abstract}
A novel process is demonstrated whereby dense arrays of single-walled carbon nanotubes (SWNT) are grown directly at the interface of a carbon material or carbon fiber. This growth process combines the concepts of SWNT tip growth and alumina-supported SWNT base growth to yield what we refer to as "odako" growth. In odako growth, an alumina flake detaches from the carbon surface and supports catalytic growth of dense SWNT arrays at the tip, leaving a direct interface between the carbon surface and the dense SWNT arrays. In addition to being a new and novel form of SWNT array growth, this technique provides a route toward future development of many important applications for dense aligned SWNT arrays.
\end{abstract}

\section{KEYWORDS}

Carbon nanotubes, carbon fibers, chemical vapor deposition

\section{Introduction}

The discovery that carbon nanotubes (CNT), and singlewalled carbon nanotubes (SWNT) in particular, can be grown in dense, aligned arrays [1-3] has inspired a continuous flow of innovative discoveries with great potential to substantially impact important future applications. Among these applications are supercapacitors [4], field emitters [1], transparent and aligned conductive films [5], adhesive tapes [6], membrane filters [7], and loudspeaker devices [8], among others. The use of these so-called super-growth processes to rapidly produce ultra-long, aligned SWNTs is continuing to inspire new, important applications. Nonetheless, the drawback of the growth process for direct integration of as-grown, aligned SWNT materials into many applications is the requirement of a thin oxide layer between the catalyst and the growth substrate to support the catalyst formation and activity. Although simple and efficient techniques have been developed to transfer aligned SWNTs to host surfaces [5], the most attractive situation involves a growth process where the catalytic activity takes place directly at the interface of a desired material, such as a carbon or conducting surface. Despite the exciting mechanical and electrical properties of SWNTs combined with the as-aligned, ultra-long SWNT materials grown in the water-assisted super-growth process, the sensitivity of the growth process to the alumina catalyst support severely limits the potential applications of these materials.

Address correspondence to hauge@rice.edu 
As a result, there has been a substantial interest in the growth of CNTs without the presence of an oxide support, and directly on the surface of interest. Of all materials, carbon materials are the most exciting since the strong, lightweight nature of carbon fibers, in particular, make them a primary component of multifunctional composite materials well-equipped for use in the aerospace industry. Due to this, there has been an extensive effort to grow multi-walled nanotubes (MWNT) and nano-fibrillar carbon structures on carbon materials [9-14], with only a few recent studies, however, reporting high density MWNT growth. An alumina layer [9] or an $\mathrm{SiO}_{2}$ [10] layer between the carbon surface and nanotubes is a necessary component to achieve reasonable growth. Direct growth on carbon surfaces [11-14] results in sparse nanotubes grown with low yields in concert with a surface potentially damaged by the catalyst layer. A growth process which results in high density SWNT growth that preserves an SWNT/carbon fiber interface is demonstrated for the first time in the work presented in this study.

In this work we present first results depicting a form of growth that combines aspects of tip, or "kite" growth [15], and the typical base growth achieved in the water-assisted super-growth technique by growing dense, aligned SWNTs from the tips of fibrils supporting thin, flexible oxide flakes. The result is what we call "odako" growth, where odako is a Japanese word which literally means "giant kite $^{\prime 1}$. This name was chosen since dense SWNT structures are grown from a flake of alumina (broken from a continuous, deposited layer) which lifts away from the growth substrate supporting many thousand catalyst particles on its underside growing SWNTs. The SWNTs remain attached to the growth substrate, tethering down the flake. The result of this growth process is a material where SWNTs reside at the interface of the growth substrate, while the oxide layer and catalyst remain constantly exposed to the reaction gas flow at the top. Here, we detail the aspects of this novel growth technique, and emphasize applications and processes which may be achieved based upon this process for aligned SWNT growth.

\section{Experimental}

In order to achieve the growth reported in this study, we utilize low-pressure (1.4 Torr) water-assisted growth conditions with atomic hydrogen catalyst activation, as described in detail elsewhere [1618]. This involves exposure to atomic hydrogen, produced via a tungsten hot filament, for $30 \mathrm{~s}$ in the presence of $\mathrm{H}_{2}$ prior to growth following rapid sample insertion into a pre-heated furnace. In order to grow dense SWNT arrays, a flow of 400 standard cubic centimeters per minute (sccm) $\mathrm{H}_{2}, 2 \mathrm{sccm} \mathrm{H}_{2} \mathrm{O}$, and $2 \mathrm{sccm} \mathrm{C}_{2} \mathrm{H}_{2}$ were utilized at a temperature of 750 ${ }^{\circ} \mathrm{C}$, as this provides the optimal conditions for SWNT growth in this process. In some cases, an elevated pressure (25 Torr) was utilized to increase the $\mathrm{C}_{2} \mathrm{H}_{2}$ partial pressure and the total decomposition rate on the catalyst (and hence, growth rate). This yields faster growth of the SWNT arrays at the expense of the formation of some double and few-walled carbon nanotubes amidst the SWNT population [18]. Nonetheless, the utilization of a low-pressure CVD system is geared toward a slower growth process than the well-known atmospheric super-growth, as the active carbon source is only abundant with partial pressures of a few mTorr. This results in a typical SWNT array growth of $\sim 100$ microns at 1.4 Torr instead of thicker arrays grown at higher pressures.

The typical process by which odako growth takes place is illustrated in Fig. 1. In order to achieve odako growth, an ultra-thin catalyst layer $(\mathrm{Fe}) 1 \mathrm{~nm}$ thick was deposited directly onto a carbon surface via e-beam. Two types of carbon surfaces, grafoil and carbon fibers, were utilized for this study. Directly following the deposition of the catalyst layer, an overlayer of alumina $\left(\mathrm{Al}_{2} \mathrm{O}_{3}\right) 5 \mathrm{~nm}$ thick was deposited onto the catalyst. Our recent studies focusing on imaging the morphology of thin catalyst

\footnotetext{
${ }^{1}$ Odako is derived from the Japanese word dako, meaning kite. Annual traditional festivals are held in Japan where these giant kites, often manned by hundreds of people, are flown. One can retrieve several pictures of odakos in a simple web search. A typical picture of an odako can be found here: http://farm1.static.flickr.com/164/359023438_0e376a7240.jpg?v=0, accessed 3-13-09.
} 
films emphasize that catalyst deposition results in the formation of Fe islands due to the balance of the catalyst-surface interfacial interaction relative to the intra-particle atomic interactions [19]. This means that the deposition of the alumina overlayer results in the formation of a continuous film of alumina that not only interacts with the deposited catalyst islands, but also with the carbon surface. Upon rapid insertion into the hot furnace in the presence of atomic hydrogen, the alumina layer will crack into small flakes - a feature inherent to a brittle e-beamed amorphous alumina layer - and carbon growth will be catalyzed from the exposed edges. Lift-off of the alumina flake will occur due to the volatility of the alumina-carbon interface ( $\mathrm{C}-\mathrm{O}$ bond) in the presence of hydrogen, which will hydrogenate the $\mathrm{C}$ species and detach the $\mathrm{Al}_{2} \mathrm{O}_{3}$ flake. Since detachment occurs in the presence of carbon, SWNT nucleation occurs while the catalyst particle is at the interface of the carbon surface, leaving the first carbon precipitates "molded" by the carbon surface and likely allowing some $\mathrm{sp}^{3}$ carbon bond formation between SWNTs and the carbon surface. This appears to tether down the dense SWNT array while the flake supporting the catalyst is constantly exposed to the incoming $\mathrm{C}_{2} \mathrm{H}_{2}$ feedstock. This process is illustrated schematically in Fig. 1, and will be depicted experimentally in images presented in the next section.

\section{Results and discussion}

A high magnification scanning electron microscope (SEM) image, shown in Fig. 2, demonstrates the concept of odako growth of dense SWNT arrays on a grafoil surface. As demonstrated in Fig. 1, odako growth involves the detachment of $5 \mathrm{~nm}$ thick flakes of alumina which support the catalyst islands responsible for the growth of the dense SWNT arrays. This is evident in Fig. 2, as both the alumina flake and the catalyst particles on the underside (inset) of the flake are visible. The thin nature of the flake in Fig. 2 results in a level of transparency that allows for the imaging of the catalyst layer on the underside. This is not surprising since we recently reported flakes of $40 \mathrm{~nm}$ thickness that were also transparent in SEM images [17]. The inset image in Fig. 2 (scale

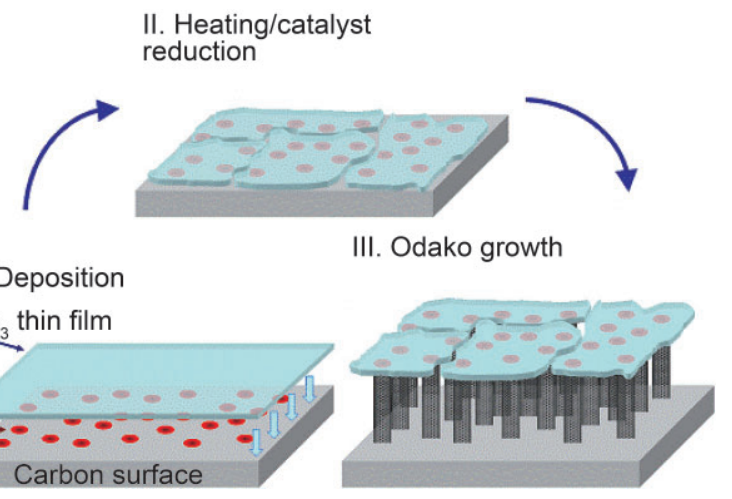

Figure 1 Illustration of the odako growth process, starting from catalyst deposition, heating, and then growth

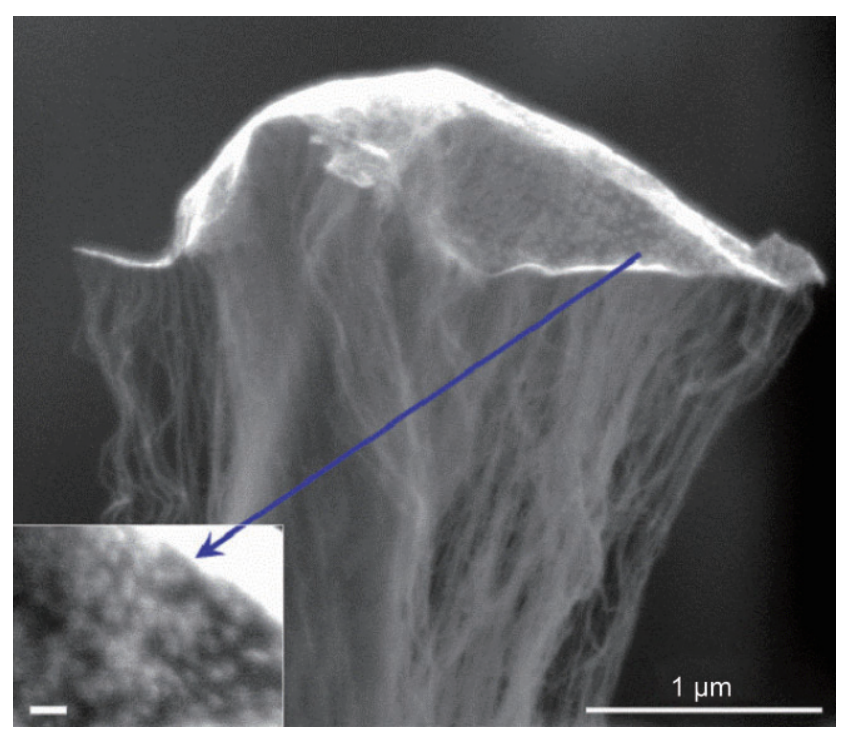

Figure 2 SEM image of an individual fibril made by the odako growth process. Inset is a high-magnification image of the flake showing the catalyst particles (scale bar $=50 \mathrm{~nm}$ )

bar=50 nm) demonstrates a higher magnification view of the catalyst layer, which emphasizes the growth of oxide catalyst particles having a size distribution consistent with previous transmission electron microscope (TEM) imaging experiments [19]. It should be noted that X-ray photoelectron spectroscopy (XPS) characterization of a homogenous layer of odako fibrils grown from a grafoil surface indicated the clear presence of $\mathrm{Fe}$ and $\mathrm{Al}_{2} \mathrm{O}_{3}$ residing at the tips of the fibrils following growth (see Electronic Supplementary Material (ESM)). Since the penetration depth of electrons ejected by incoming $\mathrm{X}$-rays is not likely to be deeper than $10 \mathrm{~nm}$ into the surface, the presence of a substantial amount of catalyst (especially in the surface geometry 
demonstrated by the SEM image shown in the ESM) is further proof that the $\mathrm{Fe}$ is adhering to the alumina flake during lift-off and is responsible for the growth of the SWNT arrays. This is not surprising, since the growth of dense SWNT arrays via the classical base growth mechanism is inherently dependent on the presence of an alumina surface to support the catalyst layer. Therefore, our ability to grow long, dense arrays of SWNTs is a principal indication that the catalyst is supported by the alumina flakes positioned at the tips of the fibrils. As will be discussed at a later point, control experiments where $1 \mathrm{~nm}$ of Fe is deposited onto a carbon fiber surface resulted in no measurable carbon nanotube growth, further supporting our model for odako growth. In addition, it is also interesting to note that the appearance of the flake in Fig. 2 emphasizes its flexible nature, allowing it to fold over and appear as a "cap" on the growing SWNT array. This is a feature inherent to such a thin oxide layer, and opens up interesting possibilities for nanoscale manipulation of catalyst layers.

Further SEM images, presented in Fig. 3, illustrate the concept of odako growth on a grafoil surface both with growth carried out at low pressure (1.4 Torr) for 15 min (Figs. 3(a) and 3(b)), and at elevated pressures (25 Torr) for 30 min (Figs. 3(c) and 3(d)). In general, we note that the odako growth process takes place with the same efficiency as typical super-growth, resulting in uniform and dense array growth over the complete surface. The primary difference between the two is the formation of alumina flakes supporting the catalyst, resulting in the growth of individual, compact fibrils of SWNTs instead of a bulk array. From the SEM images depicting odako growth at elevated pressures (Figs. 3(c) and 3(d)), it is evident that odako growth occurs uniformly over the whole surface. Higher magnification images of these fibrils (Fig. 3(d)) indicate growth via the same mechanism as presented in Fig. 1, with an evident layer of alumina capping the exposed ends of the SWNT array. In all images obtained of this growth process, it is evident that, regardless of the length or size of the fibril, it always appears anchored to the carbon surface. Despite our efforts, it should be noted that the level of adhesion between the fibrils and the carbon surface is difficult to test in any welldefined way. Utilizing an adhesive to contact a thick layer of fibrils (as shown in Fig. 3(c)), where there is no carbon surface exposed to the adhesive, we find that removal of the adhesive also detaches layers of

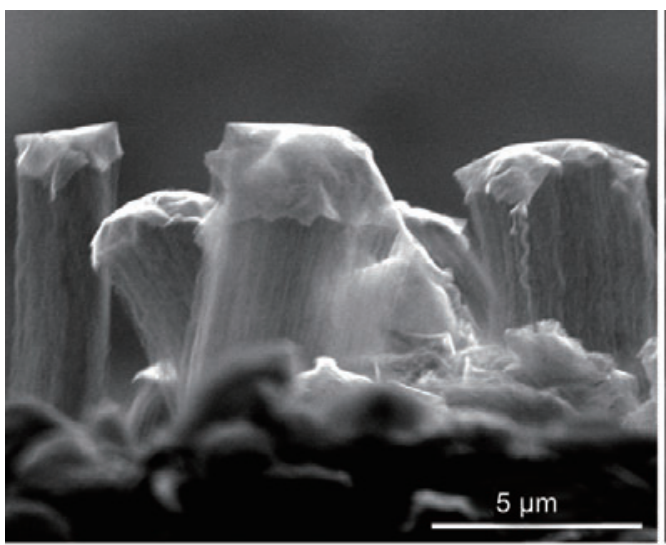

(a)

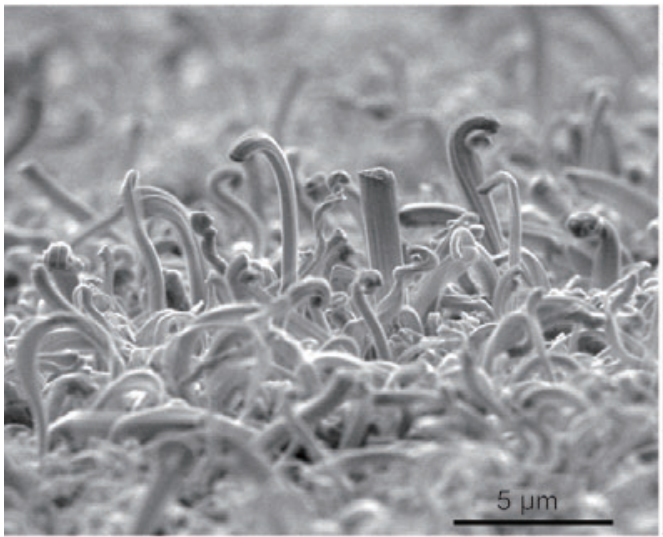

(c)

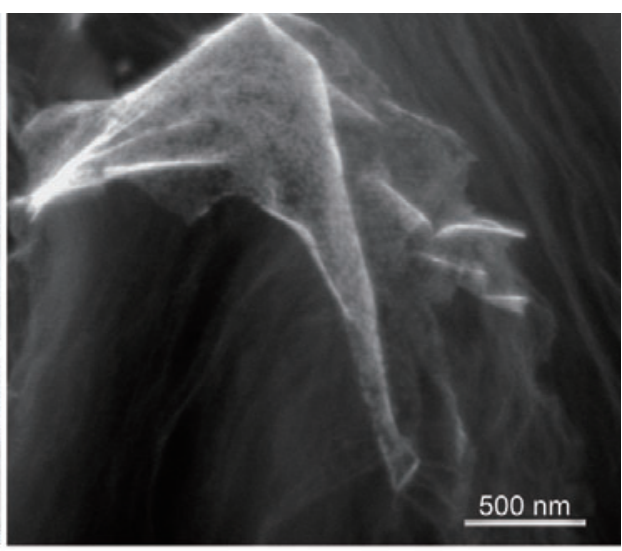

(b)

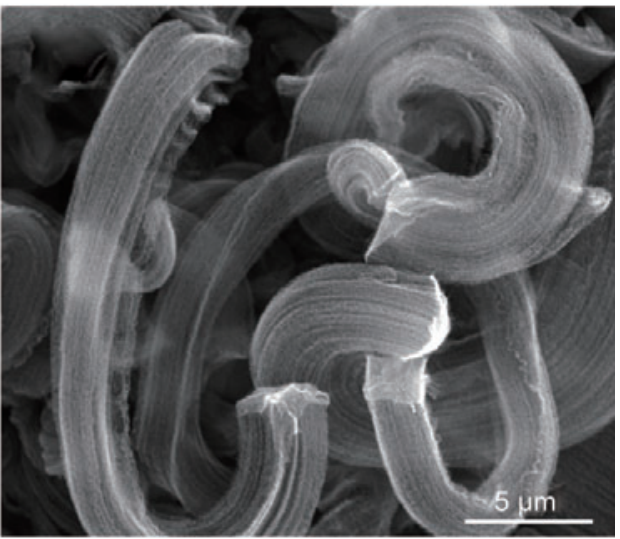

(d)
Figure 3 SEM images of SWNT fibrils produced via the odako growth process for low pressure (1.4 Torr, (a) and (b)), and high pressure (25 Torr, (c) and (d)) growth conditions 
graphite from the grafoil, leaving the grafoil-SWNT interface intact. In addition, in tests where the surface was wetted with a solvent (ethanol) and allowed to dry, capillary force-induced drying of the SWNTs appeared to leave the interface between the fibril and the carbon surface intact, despite the densification of the fibrils and mechanical stresses in the layer due to interfibril interactions. Therefore, we emphasize that the fibrils qualitatively appear well attached to the carbon surface, which could be a result of a nucleation phase occurring at the interface of the carbon surface-allowing the cap formation typical in nucleation to result in a carbon precipitate that involves $\mathrm{C}-\mathrm{C}$ bonds to the carbon growth substrate. However, this is speculative and is a focus of ongoing work utilizing composite materials made from odako growth on carbon surfaces for mechanical testing studies.

In order to investigate the nanotubes that are being grown by this technique, Raman spectroscopy (Fig. 4) and TEM (Fig. 5) were carried out. From the Raman spectroscopy data, a relatively low intensity of the $\mathrm{D}$ band with respect to that of the $G$ band that is typically evident during SWNT growth is also evident in Fig. 4(a). The G/D ratio for $514 \mathrm{~nm}$ excitation is $\sim$, for 633 $\mathrm{nm}$ excitation is $\sim 10.8$, and for $785 \mathrm{~nm}$ excitation is $\sim 3.5$. The higher intensity D-band at lower excitation energy is likely due to the presence of some amorphous carbon. In addition, the broad range of diameter-dependent

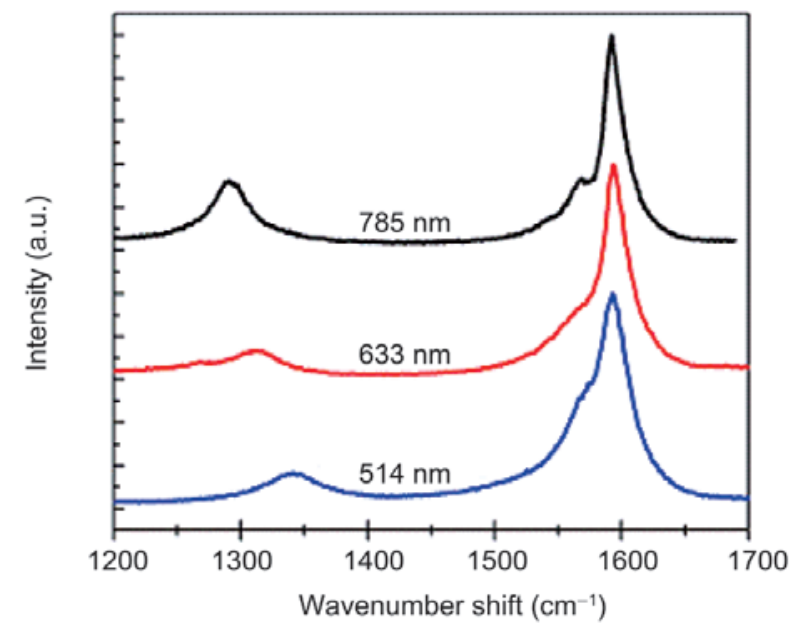

(a) radial breathing modes (RBM) at all three excitation energies (Fig. 4(b)) emphasizes a broad diameter distribution of SWNTs. Comparison of the three single laser-line spectra in Fig. 4(b) to a resonant Raman map of similar super-growth material constructed by Doorn et al. [20] indicates similar features. In particular, the lowest energy excitations indicate the presence of a family of $\mathrm{E}_{11}$ metallic SWNT transitions $\left(\sim 150 \mathrm{~cm}^{-1}\right)$, and a broad range of $E_{11}$ and $E_{22}$ semiconducting SWNT transitions $\left(60-130 \mathrm{~cm}^{-1}\right)$ that emphasize the presence of SWNTs having diameters greater than $3 \mathrm{~nm}$. Also present are a family of small diameter SWNTs $(d<1.5$ $\mathrm{nm}$ ) evident from RBMs at frequencies higher than 190 $\mathrm{cm}^{-1}$. Furthermore, excitation at $633 \mathrm{~nm}(1.96 \mathrm{eV})$ further emphasizes the presence of some very small diameter SWNTs $(<0.9 \mathrm{~nm})$ and also the signature of metallic and semiconducting SWNTs from families present in the lower energy excitation spectra. TEM imaging also confirms this general picture, as shown in Fig. 5. In order to prepare the TEM sample, the SWNTs were bath sonicated in ethanol for $15 \mathrm{~min}$, and drop dried onto a TEM grid. The presence of many SWNTs is visible through close inspection of a representative TEM image shown in Fig. 5(a). Analysis of this image confirms the presence of a slight majority of fairly large diameter (2 $-4 \mathrm{~nm})$ SWNTs, with some smaller (1-2 nm) and even larger $(4-6 \mathrm{~nm})$ SWNTs as well. It should be noted that the smaller diameter SWNTs consistently appear highly bundled in TEM images, resulting in difficulty in

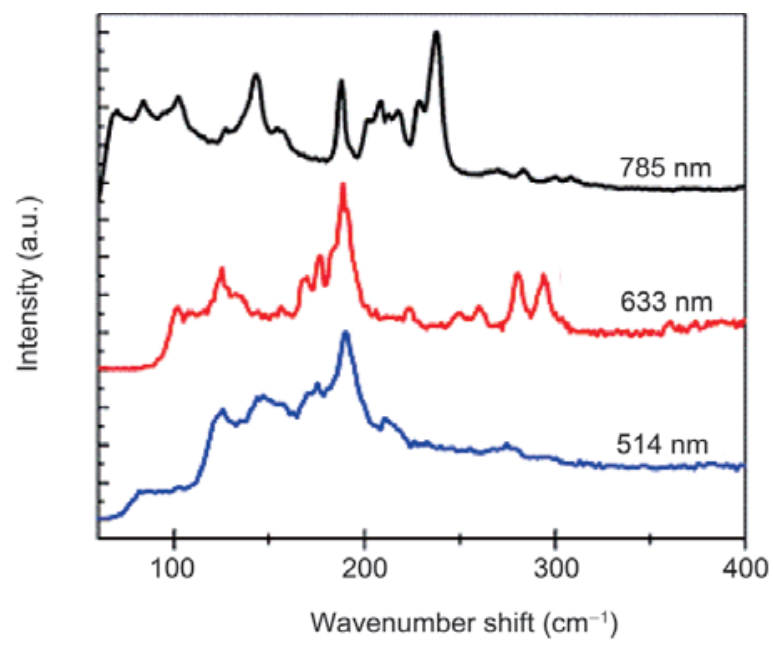

(b)

Figure 4 Raman spectra of aligned carbon nanotube fibrils formed through the odako growth process: (a) D and G bands, and (b) radial breathing modes with excitation at three laser lines between 514 and $785 \mathrm{~nm}$ 
identifying them properly amidst their larger diameter counterparts that remain unbundled. To better clarify their presence, arrows are placed next to spots where cross-sectional views of small diameter SWNTs in bundles are located. In addition, it should be noted that higher resolution images also emphasize the presence of a few nanotubes with 2-3 walls, even though this is typically only found to occur with nanotubes having diameters greater than $\sim 5.5-6 \mathrm{~nm}$ in diameter. The general SWNT diameter distribution observed by TEM is consistent with previous reports utilizing this growth

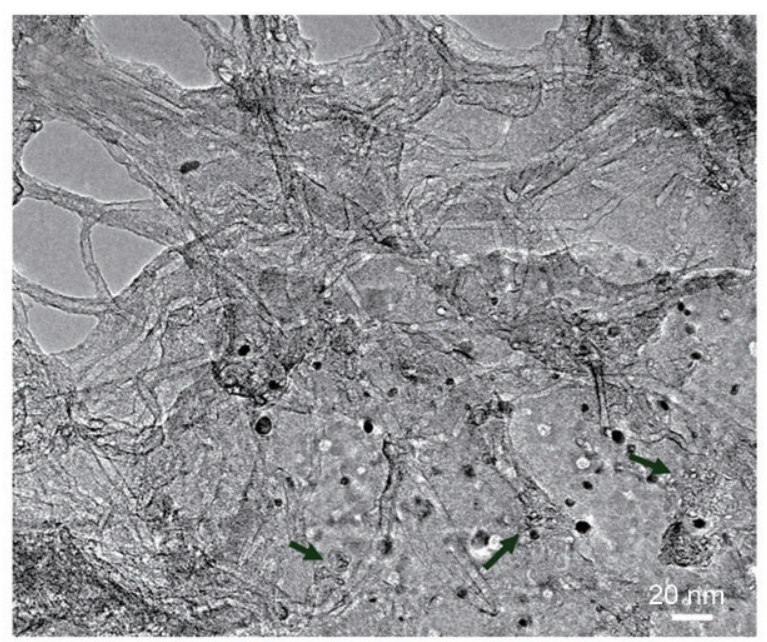

(a)

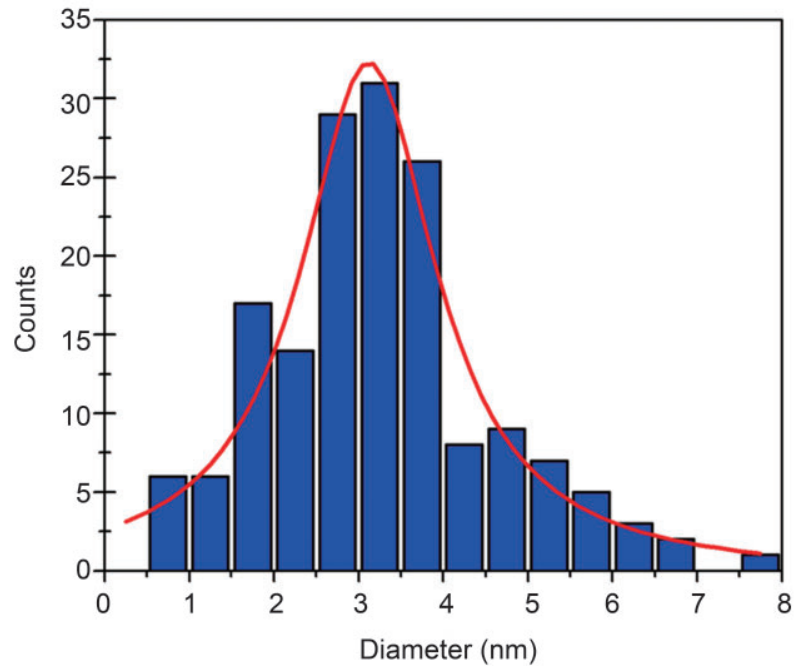

(b)

Figure 5 (a) Representative TEM image showing the many SWNTs that are typically present in the odako growth process (both individual and bundled). Arrows are pointing toward regions where a cross-sectional view of bundled small diameter SWNTs is visible; (b) diameter distribution of SWNTs as determined by multiple TEM images after sampling diameters of 164 SWNTs in total technique [18-21]; a distribution of SWNT diameters from sampling of multiple TEM images is shown in Fig. 5(b). It should be noted that this is only a crude estimate, since effects such as small diameter SWNT bundling can significantly alter the measured diameter distribution. Nonetheless, the diameter distribution appears to fit a Lorentzian curve having a peak center located at $\sim 3.1 \mathrm{~nm}$. This is consistent with other reports utilizing the alumina supported, water-assisted growth technique and further emphasizes the similarity between the SWNT material obtained in this process and that in the classic base-growth process.

In addition to growth on grafoil surfaces, we also demonstrate the concept of odako growth on carbon fiber surfaces. Since carbon fibers are principal components of lightweight composite materials utilized extensively in aerospace applications, carbon fibers represent a an interesting prototype material for demonstration of the odako growth process. Shown in Fig. 6 is a series of SEM images depicting the same growth process as that previously discussed on the surface of individual carbon fibers. In this case, the catalyst layer and oxide support were deposited directly onto a weave of carbon fiber mesh material (see ESM). As a result, the top layer of fibers exposed to the e-beam source are coated with catalyst and oxide, with some fibers in the inner portion of the material (and under the weaved portions) uncoated. The images presented in Fig. 6 correspond to those in Fig. 3, with products of low pressure growth being shown in Figs. 6(a) and 6(b), and products of growth at elevated pressures being shown in Figs. 6(c) and 6(d). From the images in Fig. 6(a) and 6(b), it is evident that the odako growth process occurs in the same fashion as discussed previously when carbon fibers are utilized as the growth substrate. The uncoated part shown on the left side in Fig. 6(a) represents a region where the weave of carbon fiber mesh on the coated material results in a portion of the fibers being covered during deposition, and hence left uncoated by catalyst. Nonetheless, it is again evident that the odako growth process occurs uniformly over the carbon fiber surface. The higher magnification SEM image in Fig. 6(b) emphasizes the presence of alumina flakes at the end of the SWNT fibrils, which retain a cylindrical morphology mimicking the geometry of

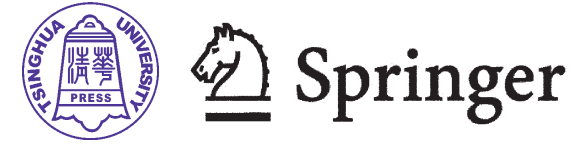




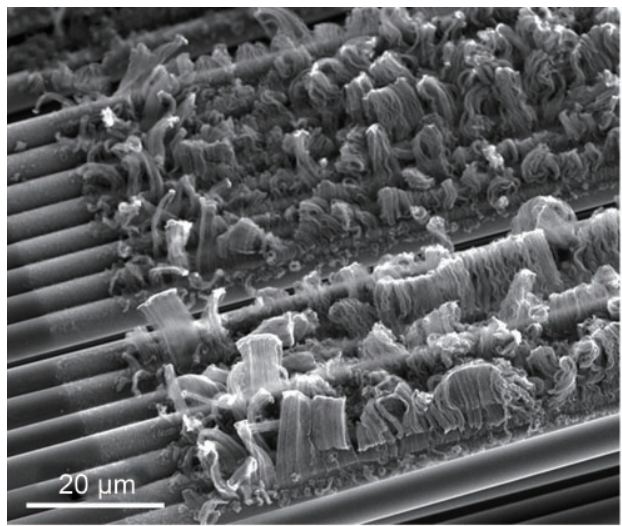

(a)

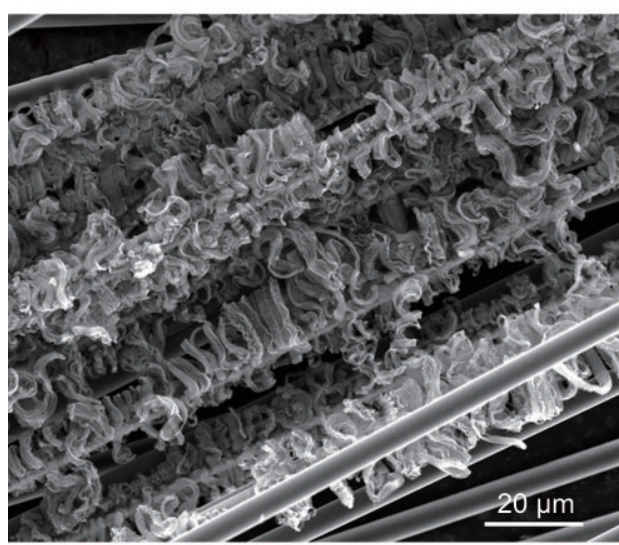

(c)

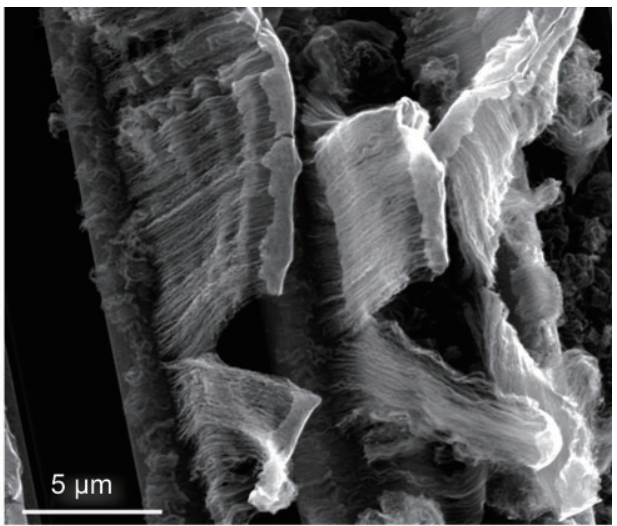

(b)

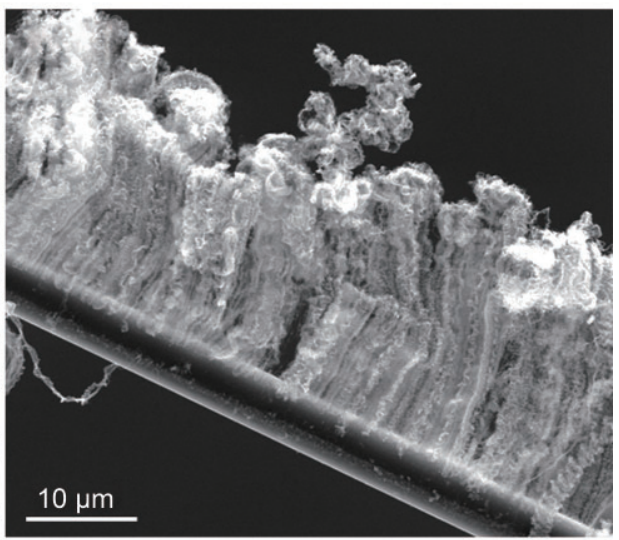

(d)

Figure 6 SEM images of SWNTs grown via the odako growth process on carbon fiber surfaces at 1.4 Torr (a) and (b), and 25 Torr (c) and (d)

the carbon fiber surface. It should be noted that with the line-of-sight technique for catalyst deposition employed here (e-beam), only one side of the fiber can be coated with catalyst and oxide. Nonetheless, advances in the ability to coat three-dimensional surfaces with metal, such as in atomic layer deposition (ALD) or by dip-coating processes, provide a route where this growth technique can be utilized for the complete coverage of a three-dimensional surface of an object such as a carbon fiber.

Further SEM images shown in Figs. 6(c) and 6(d) emphasize the more efficient growth of longer fibrils at elevated pressures from carbon fiber surfaces. For many applications, such as adhesion layers in composite materials, a long fibril may not be necessary to achieve good microscopic load transfer between fibers and the polymer matrix. Nonetheless, as we demonstrate here, longer fibrils can be achieved through the water-assisted growth process carried out at elevated pressures. The SEM image shown in
Fig. 6(d) also emphasizes the lack of residual alumina on the carbon fiber itself, since this would clearly appear as bright spots on the surface of the carbon fiber if it remained. This result was invariably found during imaging odako growth, as all the deposited alumina appears to be present at the tips of the growing fibrils and absent at the interface with the carbon fiber surface. Higher magnification imaging of the carbon fiber surface also does not indicate any significant presence of residual $\mathrm{Fe}$, whereas $\mathrm{Fe}$ is observed on the underside of the flake (Fig. 2).

In addition, we demonstrate the ability to perform odako growth on a full strand of the carbon fiber mesh material, as shown in Fig. 7. On the righthand side of the image is a strand of the carbon fiber weave, taken from the mesh material on which catalyst was deposited (see ESM), prior to growth. On the left-hand side of the image is a similar strand of carbon fiber material, following growth at $750{ }^{\circ} \mathrm{C}$ at elevated pressures. The notably black regions on 


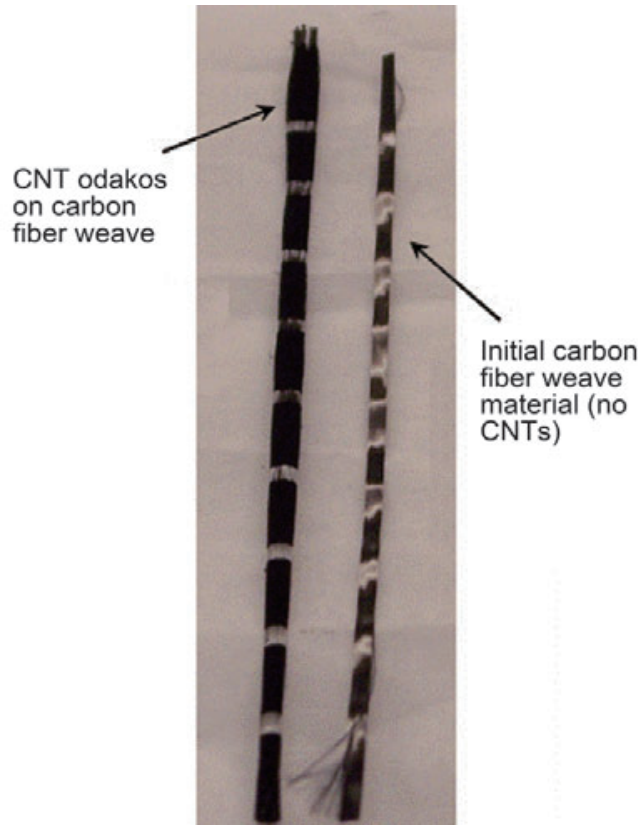

Figure 7 Photograph showing a piece of carbon fiber mesh material before (right) and after (left) the growth process. The lack of growth on some parts of the mesh on the left side is due to deposition of catalyst on a weaved fabric of this material (see ESM)

the strand on which growth took place correspond to areas where odako growth, similar to that shown in Fig. 6, has occurred. It should be noted that the stripes where no growth occurs in Fig. 7 are due to the weaved nature of the material on which catalyst deposition took place (i.e., no catalyst is deposited under weaved sections). The ability to achieve odako growth over the whole strand (where catalyst resides) emphasizes the large-scale nature of this growth process, similar to the vertical array growth obtained by water-assisted super-growth.

Finally, in closing, it should be noted that this process may not be limited to only carbon surfaces, but other novel growth substrates may also be candidates on which odako growth can occur. For example, a similar process may be achievable on quartz fibers, where $\mathrm{SiC}$ and $\mathrm{C}$ layers are grown as an overlayer on the fiber, allowing the odako growth process to result in the type of strong SWNT interface with the fiber that is sought for mechanical reinforcement applications. The same argument can be applied to a variety of metallic surfaces, where electrical contact between a conducting surface and the SWNT layer is crucial in applications such as electrolytic capacitor devices.

\section{Conclusions}

We present a novel technique, termed odako growth, for the synthesis of dense, aligned arrays of singlewalled carbon nanotubes having a preserved SWNT/ carbon surface interface, and a catalyst and oxide support that are always exposed to the reaction gas flow. We demonstrate this growth process on grafoil and carbon fiber surfaces, and emphasize the potential for this growth process on a variety of additional surfaces.

\section{Acknowledgements}

The authors thank D. Natelson for use of equipment for catalyst evaporation. Also, special thanks to C. Kittrell, S. Ripley, and R. Wahi for discussions.

Electronic Supplementary Material: Supplementary material is available in the online version of this article at http://dx.doi.org/10.1007/s12274-009-9050-7 and is accessible free of charge.

\section{References}

[1] Fan, S. S.; Chapline, M. G.; Franklin, N. R.; Tombler, T. W.; Cassell, A. M.; Dai, H. Self-oriented regular arrays of carbon nanotubes and their field emission properties. Science 1999, 283, 512-514.

[2] Murakami, Y.; Chiashi, S.; Miyauchi, Y.; Hu, M. H.; Ogura, M.; Okubo, T.; Maruyama, S. Growth of vertically aligned single-walled carbon nanotube films on quartz substrates and their optical anisotropy. Chem. Phys. Lett. 2004, 385, 298-303.

[3] Hata, K.; Futaba, D. N.; Mizuno, K.; Namai, T.; Yumura, M.; lijima, S. Water assisted highly efficient synthesis of impurity-free single-walled carbon nanotubes. Science 2004, 306, 1362-1364.

[4] Futaba, D. N.; Hata, K.; Yamada, T.; Hiraoka, T.; Hayamizu, Y.; Kakudate, Y.; Tanaike, O.; Hatori, H.; Yumura, M.; lijima, S. Shape-engineerable and highly densely packed single-walled carbon nanotubes and their application as super-capacitor electrodes. Nat. Mater. 2006, 5, 987-994.

[5] Pint, C. L.; Xu, Y. Q.; Pasquali, M.; Hauge, R. H. Formation of highly dense aligned ribbons and

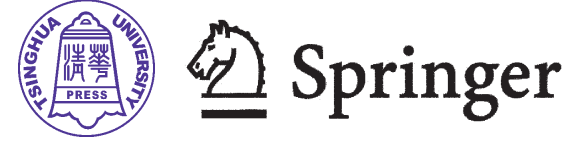


transparent films of single-walled carbon nanotubes directly from carpets. ACS Nano 2008, 2, 1871-1878.

[6] Sethi, S.; Ge, L. H.; Ci, L. J.; Ajayan, P. M.; Dhinojwala, A. Gecko-inspired carbon nanotube-based self-cleaning adhesives. Nano Lett. 2008, 8, 822-825.

[7] Majumder, M.; Chopra, N.; Andrews, R.; Hinds, B. J. Nanoscale hydrodynamics-Enhanced flow in carbon nanotubes. Nature 2005, 438, 44.

[8] Xiao, L.; Chen, Z.; Feng, C.; Liu, L.; Bai, Z. Q.; Wang, Y.; Qian, L.; Zhang, Y. Y.; Li, Q. Q.; Jiang, K. L.; Fan, S. S. Flexible, stretchable, transparent carbon nanotube thin film loudspeakers. Nano Lett. 2008, 8, 4539-4545.

[9] Liu, X.; Baronian, K. H. R.; Downard, A. J. Direct growth of vertically aligned carbon nanotubes on a planar carbon substrate by thermal chemical vapor deposition. Carbon 2009, 47, 500-506.

[10] Qu, L. T.; Zhao, Y.; Dai, L. Carbon microfibers sheathed with aligned carbon nanotubes: Towards multidimensional, multicomponent, and multifunctional nanomaterials. Smal/ 2006, 2, 1052-1059.

[11] Tzeng, S. -S.; Hung, K. -H.; Ko, T. -H. Growth of carbon nanofibers on activated carbon fiber fabrics. Carbon 2006, 44, 859-865.

[12] De Riccardis, M. F.; Carbone, D.; Dikonimos Makris, Th.; Giorgi, R.; Lisi, N.; Salernitano, E. Anchorage of carbon nanotubes grown on carbon fibres. Carbon 2006, 44, 671-674.

[13] Thostenson, E. T.; Li, W. Z.; Wang, D. Z.; Ren, Z. F.; Chou, T. W. Carbon nanotube/carbon fiber hybrid multiscale composites. J. Appl. Phys. 2002, 91, 6034-6037.

[14] Hung, K. H.; Tzeng, S. S; Kuo, W. S.; Wei, B. O.; Ko, T. $\mathrm{H}$. Growth of carbon nanofibers on carbon fabric with Ni nanocatalyst prepared using pulse electrodeposition.
Nanotechnology 2008, 19, 295602.

[15] Huang, S. M. Woodson, M.; Smalley. R. E.; Liu, J. Growth mechanism of oriented long single walled carbon nanotubes using "fast heating" chemical vapor deposition process. Nano Lett. 2004, 4, 1025-1028.

[16] Pint, C. L.; Nicholas, N.; Pheasant, S. T.; Duque, J. G.; Parra-Vasquez, A. N. G.; Eres, G.; Pasquali, M.; Hauge, R. H. Temperature and gas pressure effects in vertically aligned carbon nanotube growth from Fe-Mo catalyst. J. Phys. Chem. C 2008, 112, 14041-14051.

[17] Pint, C. L.; Pheasant, S. T.; Pasquali, M.; Coulter, K. E.; Schmidt, H. K.; Hauge, R. H. Synthesis of high aspect-ratio carbon nanotube "flying carpets" from nanostructured flake substrates. Nano Lett. 2008, 8, 1879-1883.

[18] Pint, C. L.; Pheasant, S. T.; Parra-Vasquez, A. N. G.; Horton, C.; Xu, Y. O.; Hauge, R. H. Investigation of optimal parameters for oxide-assisted growth of vertically aligned single-walled carbon nanotubes. J. Phys. Chem. C 2009, 113, 4125-4133.

[19] Amama, P. B.; Pint, C. L.; McJilton, L.; Kim, S. M.; Stach, E .A.; Murray, P. T.; Hauge, R.H.; Maruyama, B. Role of water in super growth of single-walled carbon nanotube carpets. Nano Lett. 2009, 9, 44-49.

[20] Doorn, S. K.; Araujo, P. T.; Hata, K.; Jorio, A. Excitons and exciton-phonon coupling in metallic single-walled carbon nanotubes: Resonance raman spectroscopy. Phys. Rev. B 2008, 78, 165408.

[21] Zhao, B.; Futaba, D. N.; Yasuda, S.; Akoshima, M.; Yamada, T.; Hata, K. Exploring advantages of diverse carbon nanotube forests with tailored structures synthesized by supergrowth from engineered catalysts. ACS Nano 2009, 3, 108-114. 